\title{
The Effect of Magnesium Sulphate on Intubating Condition for Rapid-Sequence Induction; Comparative Study of Magnesium Sulphate versus Ketamine in Rapid Sequence Induction
}

\author{
MOHAMED S. ABDEL AZIZ, M.D.; HEBA B. EL-SERWI, M.D. and DINA GALAL ABO-DIEF, M.Sc. \\ The Department of Anesthesia, Intensive Care and Pain Management, Faculty of Medicine, Ain Shams University
}

\begin{abstract}
Background: Rapid sequence induction is the preferred method of endotracheal intubation in the emergency setting. The rapid sequence intubation technique involves the prompt sequential administration of a predetermined dose of hypnotic agent and muscle relaxant followed by tracheal intubation within $1 \mathrm{~min}$ of giving the muscle relaxant.
\end{abstract}

Aim of Study: Test the efficacy of magnesium sulphate (MgSo4) versus ketamine to assess intubating condition (primary outcome), rocuronium onset, rocuronium duration, train-of-four ratio upon intubation, and hemodynamic variables (secondary outcomes) for rapid sequence induction.

Patients and Methods: We compared magnesium sulphate with control, ketamine with regard to intubation conditions during rapid-sequence induction. study.

Design: This is a prospective, randomized, double-blinded

Setting: The setting is at an operating room in Ain Shams University Hospital.

Patients: 75 patients scheduled for general anesthesia were randomly allocated to the following 3 groups in equal numbers.

Interventions: The control received rocuronium $0.6 \mathrm{mg} / \mathrm{kg}$; the ketamine group was given $0.5 \mathrm{mg} / \mathrm{kg}$ ketamine 2 minutes before $0.6 \mathrm{mg} / \mathrm{kg}$ rocuronium; and the magnesium group received $50 \mathrm{mg} / \mathrm{kg}$ magnesium sulphate. Intubation was initiated 50 seconds after the rocuronium injection.

Measurements: Intubating condition (primary outcome), rocuronium onset, rocuronium duration, train-of-four ratio upon intubation, and hemodynamic variables (secondary outcomes) were recorded.

Results: The excellent intubating condition was more frequent in the magnesium group ( $p$ b.05). Onset of neuromuscular block was shorter in the magnesium group than in the control, ketamine, ( $p$ b.05).

Conclusions: Magnesium sulphate pre-treatment was most likely to provide excellent intubating condition for rapid-

Correspondence to: Dr. Dina Galal Abo Dief, E-Mail: dina76795@gmail.com sequence intubation compared with the control, ketamine. However, magnesium sulphate administration is associated with a burning or heat sensation.

Key Words: Intra tracheal intubation - Magnesium sulphate - Neuromuscular block - Ketamine.

\section{Introduction}

SUCCINYLCHOLINE is the first-choice neuromuscular blocking agent for rapid-sequence intubation [1]. Succinylcholine is contraindicated in patients with major burns (beyond 48 hours), major crush injuries (beyond 48 hours), and spinal cord injuries due to the risk of hyperkalemia [2]. Several alternative methods of facilitating neuromuscular block have been introduced to improve intubating condition during rapid-sequence intubation.

Ketamine pre-treatment accelerates neuromuscular block by increasing cardiac output and blood pressure and thereby shortening rocuronium circulation time [3]. Magnesium sulphate pre-treatment [4] also provide a faster neuromuscular block than does standard dose rocuronium $(0.6 \mathrm{mg} / \mathrm{kg})$ alone. No studies have compared intubating conditions among these methods. Therefore, we compared magnesium sulphate with control, ketamine regard to intubating condition (primaryoutcome) during rapid-sequence intubation. We hypothesized that magnesium sulphate would provide an excellent intubating condition due to its profound acceleration of rocuronium onset [4]. Onset and duration of rocuronium, Train-of-Four (TOF) ratio at the time of intubation, and cardiovascular changes after rapid-sequence intubation (secondary outcomes) were also compared. 


\section{Patients and Methods}

This double-blind randomized controlled trial received approval from the Research Ethical Committee of Ain Shams University from October 2018 to April 2019. Written informed consent was obtained from all participants. Patients (age, 18-65 years; body mass index, $18.5-24.9 \mathrm{~kg} / \mathrm{m}^{2}$ ) with American Society of Anesthesiologists physical status of I-II and Mallampati class of I-II, who were scheduled for an elective operation under general anaesthesia in Ain Shams Hospital were enrolled in the study.

Exclusion criteria were renal, hepatic, cardiovascular, or neuromuscular disease; breast-feeding; pregnancy; anticipated difficult airway; and medications that influence neuromuscular transmission such as antiepileptics, corticosteroids, furosemide, and aminoglycosides [2]

After establishing pulse oximetry, electrocardiogram, and non invasive blood pressure monitoring, 75 patients were randomly allocated into 1 of 3 parallel groups (control, ketamine, and magnesium groups) in equal numbers, using a computergenerated random sequence prepared by a research assistant who was not involved in the study. The group allocation was concealed in sealed opaque envelopes that were numbered consecutively. The envelopes were opened sequentially upon patients' (consent obtained) arrival at the operating room by an anesthetist who subsequently prepared the study drugs and labeled them as" 1 st," "2nd," " 3 rd," respectively designating the order of the drug injections (Table 1).

The anesthetist who prepared the study drugs did not participate in the other process of the study and was unaware of the study objectives and outcome measures. The drugs were administered intravenously in the following sequence (Table 1) by a trained nurse who was not involved in this trial and was unaware of the treatment allocation. Magnesium sulphate $(50 \mathrm{mg} / \mathrm{kg}$ ) or the same volume of $0.9 \%$ saline (the other groups) was infused over 10 minutes. Neuromuscular monitoring was performed in agreement with the clinical research consensus (5) using TOF-Watch SX (Organon, Ltd, Dublin, Ireland). Two pediatric surface electrodes were affixed $3 \mathrm{~cm}$ apart over the ulnar nerve on the side of the wrist without either an intravenous catheter or a blood pressure cuff. The hand and forearm were immobilized.

A hand adapter (Organon) was placed on the thumb. After amidazolam $(0.05 \mathrm{mg} / \mathrm{kg})$ injection, calibration (using implanted mode 2) and stable twitches (b5\% deviation, for 2 minutes) were confirmed. Then, TOF stimulations $(2 \mathrm{~Hz}$, square wave, 200- simulus duration) were continued every 15 seconds.

One minute later, $0.5 \mathrm{mg} / \mathrm{kg}$ ketamine (ketamine group) or the same volume of $0.9 \%$ saline (the other groups) was administered. Two minutes later, $2 \mathrm{mg} / \mathrm{kg}$ propofol, $1 \mathrm{~g} / \mathrm{kg}$ fentanil, and rocuronium (control, ketamine, and magnesium group) were injected in rapid succession. The laryngoscopy was commenced 50 seconds from the start of rocuronium administration with an attempt to complete intubation within 20 seconds. A skilled staff anesthetist who was blind to the group assignments performed the intubation and graded the intubation condition. If successful intubation was not carried out within 30 seconds (ie, 70 seconds after the start of rocuronium injection), the attempt was recorded as a failure. Intubating condition (Table 2) [5]; Cormack-Lehane grade [6]; time from start of rocuronium injection $(0.6 \mathrm{mg} / \mathrm{kg})$, until TOF count reached 0 (onset of rocuronium) and time until TOF count recovered to 2 (duration of rocuronium); and TOF ratio at the time point of intubation were recorded. Mean arterial pressure and heart rate were measured at baseline; after anesthetic induction (before intubation); immediately after intubation; and at 1, 2, 3, 4, and 5 minutes after intubation.

Table (1): Order of drug injections.

- 1st study drug 2nd study drug 3rd study 4th study drug.

- Control 0.9\% Saline $0.9 \%$ Saline $0.9 \%$ Saline Rocuronium $0.6 \mathrm{mg} / \mathrm{kg}$.

- Ketamine $0.9 \%$ Saline $0.9 \%$ Saline Ketamine $0.5 \mathrm{mg} / \mathrm{kg}$ Rocuronium $0.6 \mathrm{mg} / \mathrm{kg}$.

- Magnesium Magnesium 50mg/kg 0.9\% Saline 0.9\% Saline Rocuronium $0.6 \mathrm{mg} / \mathrm{kg}$.

- 1st study drug was infused over 10 minutes; 2nd study drug was injected after both the 1 st drug administration and calibration of TOF-Watch Sx was.

- Completed; ${ }^{3 \text { rd }}$ study drug was given 1 minute after 2 nd study drug injection; and 4th study drug was administered 2 minutes after 3rd study drug injection.

Table (2): Assessment of intubating condition a [5]

- Assessed variables excellent good poor.

- Laryngoscopy easy fair difficult.

- Jaw relaxation relaxed not fully relaxed poorly relaxed.

- Resistance to blade no resistance slight resistance active resistance.

- Position of vocal cord abducted intermediate/moving closed.

- Diaphragmatic movements or cough none on 2 weak movements for b5 $\mathrm{s} 2$ movements for $\mathrm{N} 5 \mathrm{~s}$.

Excellent : All variables are excellent.

Good : All variables are either excellent or good.

Poor : A single variable is evaluated as poor.

a : Intubating conditions. 


\section{Sample size and statistics:}

Based on pilot data of 7 patients in each group, $a \geq 50 \%$ increase in the incidence of excellent intubating condition in the magnesium group compared with the other groups was expected. A sample size of 10 per group was required to detect a $50 \%$ difference in excellent intubating condition between the magnesium and the other groups with a 2 -sided test using $\alpha=.05$ and $\beta=.2$, allowing for a $10 \%$ dropout.

Intubating condition was compared using the $\chi^{2}$ or Fisher exact tests as indicated. Analysis of Variance (ANOVA) with a Bonferroni post hoc analysis or the Kruskal-Wallis test (for nonnormal distribution) was used to compare onset time, rocuronium duration, TOF ratio, and Cormack grade. Mean arterial pressure and heart rate were analyzed with repeated-measures ANOVA. Data are given as number (percentage), mean $\pm \mathrm{SD}$, or median (interquartile range) (for non normal distribution). SPSS 18.0 (SPSS Inc., Chicago, Illinois, USA). Was used for statistical analyses. A $p$-value $<0.05$ was considered significant.

\section{Results}

A total of 75 patients were enrolled from October 2018 until April 2019, and 5 patients dropped out for various reasons; thus, data from 70 patients were analyzed. The patient characteristics are summarized in (Table 3). All patients were successfully intubated. The Cormack-Lehane grade was comparable among the groups ( $p=.0 .962)$, (Table 3 ). The magnesium group had the highest incidence of excellent intubating condition compared with the other groups ( $p$ b.05). The onset time in the magnesium group (Table 4) was shorter than that in the control and ketamine ( $p$ b.05). No significant difference was observed for rocuronium duration among the 3 groups. Significant time effects ( $p$ b.001) were observed by repeatedmeasures ANOVA for mean arterial pressure and heart rate, indicating that hemodynamic variables changed over time Fig. (1). After intubation, the control group showed higher mean arterial pressure and heart rate, and the magnesium group had lower mean arterial pressure, compared with the other groups. However, no significant differences were observed in the hemodynamic variables among the groups (mean arterial pressure, $p=.28$; heart rate, $p=.18$ ). Five patients had a burning or heat sensation during the magnesium sulphate infusion, which did not require treatment. No respiratory difficulties, ptosis, or aspiration after administration of the rocuronium control dose were observed.
Table (3): Patients' characteristics.

\begin{tabular}{|c|c|c|c|}
\hline $\begin{array}{l}\text { Demographic } \\
\text { data }\end{array}$ & $\begin{array}{c}\text { Group A: } \\
\text { Magnesium } \\
\text { sulphate }(\mathrm{n}=25)\end{array}$ & $\begin{array}{l}\text { Group B: } \\
\text { Ketamine } \\
(\mathrm{n}=25)\end{array}$ & $\begin{array}{c}\text { Group C: } \\
\text { Rocuronium } \\
(\mathrm{n}=25)\end{array}$ \\
\hline $\begin{array}{l}\text { Age (years): } \\
\text { Mean } \pm \mathrm{SD} \\
\text { Range }\end{array}$ & $\begin{array}{l}37.24 \pm 10.76 \\
20-62\end{array}$ & $\begin{array}{l}38.88 \pm 6.33 \\
24-48\end{array}$ & $\begin{array}{l}36.08 \pm 5.12 \\
25-47\end{array}$ \\
\hline $\begin{array}{l}\text { Sex: } \\
\quad \text { Female } \\
\text { Male }\end{array}$ & $\begin{array}{l}8(32.0 \%) \\
17(68.0 \%)\end{array}$ & $\begin{array}{l}10(40.0 \%) \\
15(60.0 \%)\end{array}$ & $\begin{array}{l}9(36.0 \%) \\
16(64.0 \%)\end{array}$ \\
\hline $\begin{array}{l}\text { Weight }(\mathrm{kg}): \\
\text { Mean } \pm \mathrm{SD} \\
\text { Range }\end{array}$ & $\begin{array}{l}53.56 \pm 6.24 \\
45-75\end{array}$ & $\begin{array}{l}52.96 \pm 8.43 \\
39-69\end{array}$ & $\begin{array}{l}54.16 \pm 6.18 \\
42-67\end{array}$ \\
\hline $\begin{array}{l}\text { Height }(\mathrm{cm}): \\
\text { Mean } \pm \text { SD } \\
\text { Range }\end{array}$ & $\begin{array}{l}156.92 \pm 5.63 \\
142-166\end{array}$ & $\begin{array}{l}154.76 \pm 11.36 \\
132-175\end{array}$ & $\begin{array}{l}154.92 \pm 12.07 \\
130-179\end{array}$ \\
\hline $\begin{array}{l}B M I\left(w t /(h t)^{\wedge} 2\right) \\
\text { Mean } \pm \mathrm{SD} \\
\text { Range }\end{array}$ & $\begin{array}{l}21.73 \pm 2.00 \\
18.3-27.5\end{array}$ & $\begin{array}{l}21.24 \pm 3.93 \\
13-29\end{array}$ & $\begin{array}{l}22.44 \pm 4.50 \\
13-32\end{array}$ \\
\hline $\begin{array}{c}\text { ASA: } \\
\text { I } \\
\text { II }\end{array}$ & $\begin{array}{l}20(80 \%) \\
5(20 \%)\end{array}$ & $\begin{array}{l}19(76 \%) \\
6(24 \%)\end{array}$ & $\begin{array}{l}17(68 \%) \\
8 \quad(32 \%)\end{array}$ \\
\hline $\begin{array}{l}\text { Cormack Lehar } \\
\text { degree: } \\
\text { I } \\
\text { IIA } \\
\text { IIB }\end{array}$ & $\begin{array}{ll}18 & (72.0 \%) \\
4 & (16.0 \%) \\
3 & (12.0 \%)\end{array}$ & $\begin{array}{ll}19 & (76.0 \%) \\
3 & (12.0 \%) \\
3 & (12.0 \%)\end{array}$ & $\begin{array}{ll}17 & (68.0 \%) \\
5 & (20.0 \%) \\
3 & (12.0 \%)\end{array}$ \\
\hline
\end{tabular}

This table shows no statistically significant difference between groups according to demographic data.

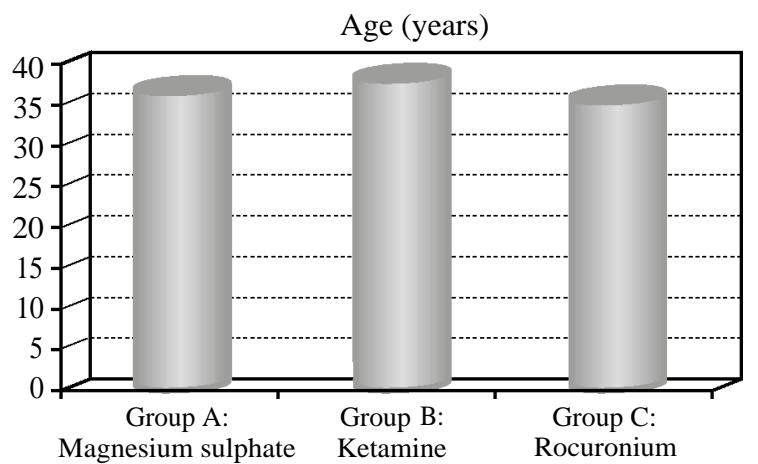

Fig. (1): Bar chart between groups according to age (years).

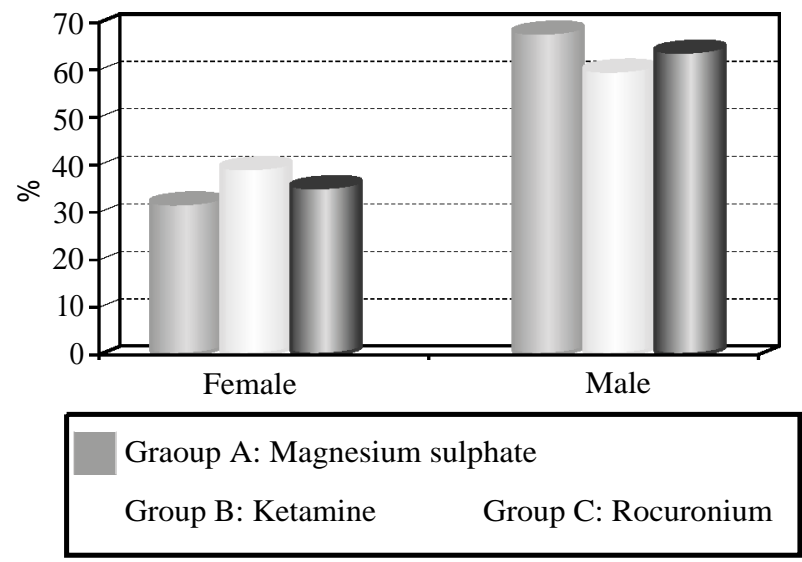

Fig. (2): Bar chart between groups according to sex. 


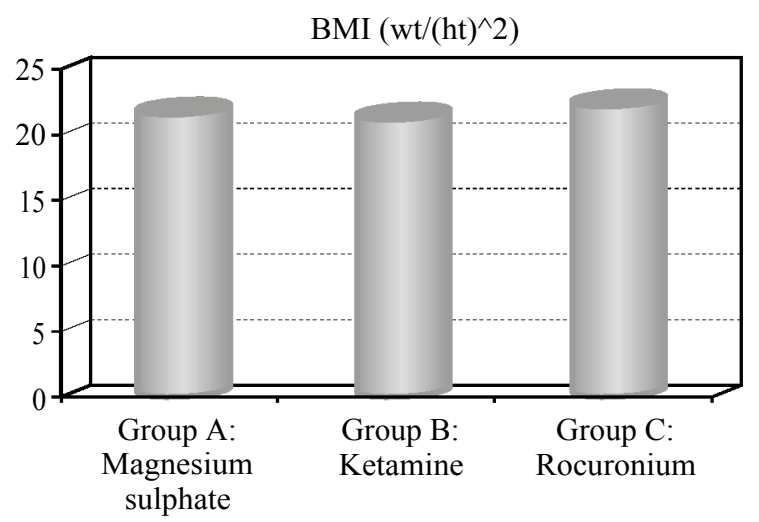

Fig. (3): Bar chart between groups according to BMI.

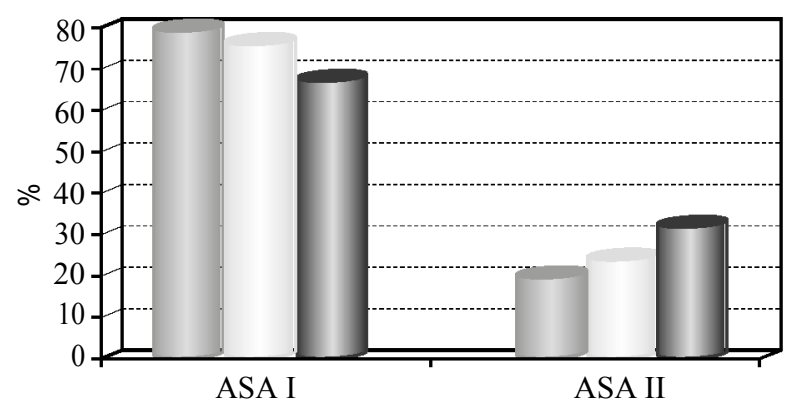

Graoup A: Magnesium sulphate

Group B: Ketamine $\square$ Group C: Rocuronium

Fig. (4): Bar chart between groups according to ASA.

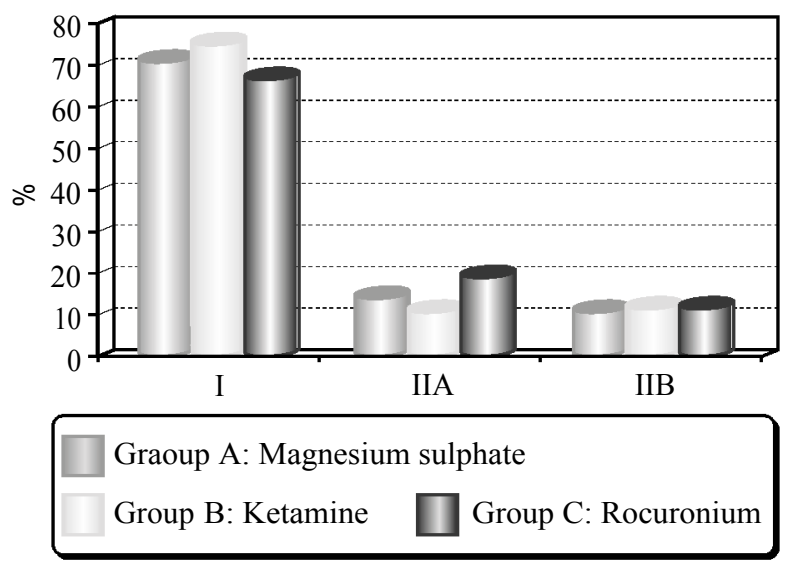

Fig. (5): Bar chart between groups according to cormack Lehane degree.

Table (4): Comparison between groups according to onset of rocuronium (sec.).

\begin{tabular}{lccccc}
\hline $\begin{array}{l}\text { Onset of } \\
\text { rocuronium } \\
(\text { sec. })\end{array}$ & $\begin{array}{c}\text { Group A: } \\
\text { Magnesium } \\
\text { sulphate } \\
(\mathrm{n}=25)\end{array}$ & $\begin{array}{c}\text { Group B: } \\
\text { Ketamine } \\
(\mathrm{n}=25)\end{array}$ & $\begin{array}{c}\text { Group C: } \\
\text { Rocuronium } \\
(\mathrm{n}=25)\end{array}$ & $\begin{array}{c}p \text { - } \\
\text { value }\end{array}$ \\
\hline Mean \pm SD & $109.33 \pm$ & $180.12 \pm$ & $183.95 \pm$ & 22.824 & $<0.001^{* *}$ \\
& 28.88 & $43.84 \dagger$ & $55.20 \dagger$ & & \\
Range & $37.63-$ & $97.82-$ & $54.71-$ & & \\
& 163.17 & 254.79 & 275.16 & & \\
\hline
\end{tabular}

This table shows statistically significant difference between groups according to onset of recuronium (sec.).

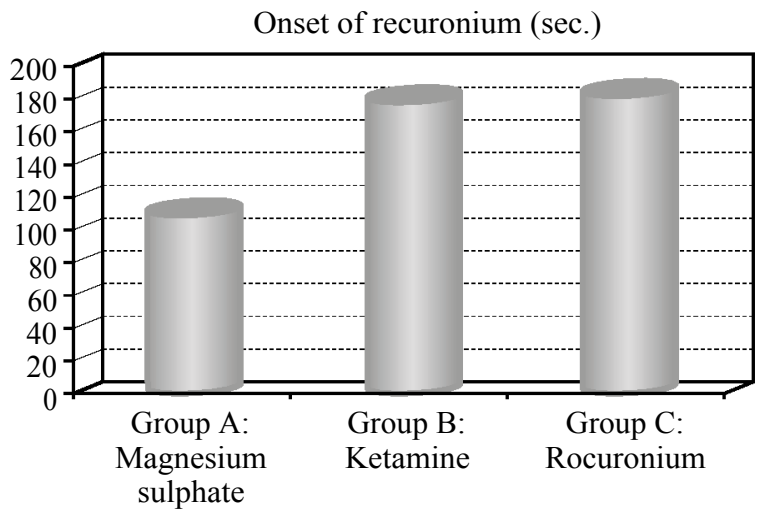

Fig. (6): Bar chart between groups according to onset of rocuronium (sec.).

Table (5): Comparison between groups according to duration of rocuronium ( $\mathrm{min})$.

\begin{tabular}{lccc}
\hline $\begin{array}{l}\text { Duration of } \\
\text { rocuronium } \\
(\text { min. })\end{array}$ & $\begin{array}{c}\text { Group A: } \\
\text { Magnesium } \\
\text { sulphate }(\mathrm{n}=25)\end{array}$ & $\begin{array}{c}\text { Group B: } \\
\text { Ketamine } \\
(\mathrm{n}=25)\end{array}$ & $\begin{array}{c}\text { Group C: } \\
\text { Rocuronium } \\
(\mathrm{n}=25)\end{array}$ \\
\hline Mean \pm SD & $\begin{array}{l}41.17 \pm 9.08 \\
\text { Range }\end{array}$ & $\begin{array}{l}38.83 \pm 14.40 \\
15.2-63.9\end{array}$ & $\begin{array}{l}37.65 \pm 15.81 \\
7.29-69.25\end{array}$ \\
\hline
\end{tabular}

This table shows no statistically significant difference between groups according to duration of rocuronium ( $\mathrm{min})$.

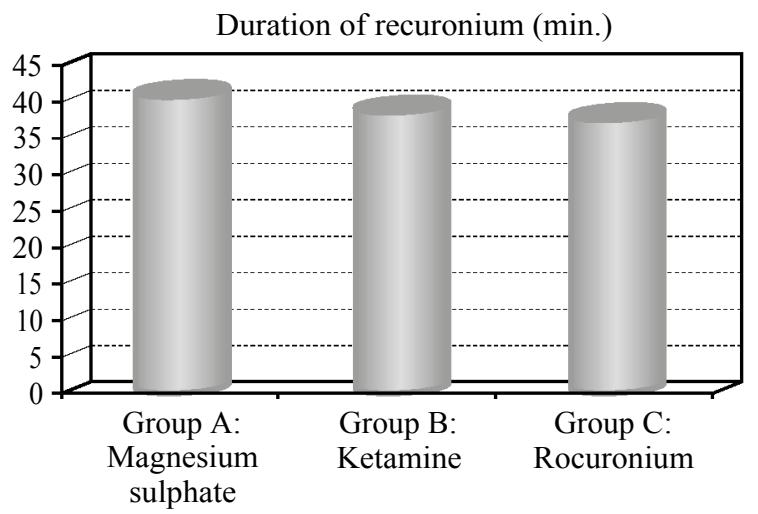

Fig. (7): Bar chart between groups according to duration of rocuronium (min).

Table (6): Comparison between groups according to TOF ratio at time point of intubation $\%$.

\begin{tabular}{lccccc}
\hline $\begin{array}{l}\text { TOF ratio at } \\
\text { time point of } \\
\text { intubation\% }\end{array}$ & $\begin{array}{c}\text { Group A: } \\
\text { Magnesium } \\
\text { sulphate } \\
(\mathrm{n}=25)\end{array}$ & $\begin{array}{c}\text { Group B: } \\
\text { Ketamine } \\
(\mathrm{n}=25)\end{array}$ & $\begin{array}{c}\text { Group C: } \\
\text { Rocuronium } \\
(\mathrm{n}=25)\end{array}$ & ANOVA & $\begin{array}{c}p- \\
\text { value }\end{array}$ \\
\hline Mean \pm SD & $84.07 \pm$ & $86.39 \pm$ & $87.88 \pm$ & 1.388 & 0.199 \\
& 14.39 & 18.46 & 10.41 & & \\
Range & $48.85-$ & $47.95-$ & $69.27-$ & & \\
& 105.33 & 118.37 & 109.83 & & \\
\hline
\end{tabular}

This table shows no statistically significant difference between groups according to TOF ratio at time point of intubation $\%$. 
Table (7): Comparison between groups according to mean arterial blood pressure ( $\mathrm{mmHg}$ ).

\begin{tabular}{lccccc}
\hline $\begin{array}{l}\text { Mean arterial blood } \\
\text { pressure }(\mathrm{mmHg})\end{array}$ & $\begin{array}{c}\text { Group A: } \\
\text { Magnesium sulphate } \\
(\mathrm{n}=25)\end{array}$ & $\begin{array}{c}\text { Group B: } \\
\text { Ketamine } \\
(\mathrm{n}=25)\end{array}$ & $\begin{array}{c}\text { Group C: } \\
\text { Rocuronium } \\
(\mathrm{n}=25)\end{array}$ & ANOVA & $\begin{array}{c}p \text { - } \\
\text { value }\end{array}$ \\
\hline $\begin{array}{l}\text { Baseline: } \\
\text { Mean } \pm \text { SD }\end{array}$ & $79.45 \pm 6.22$ & $80.77 \pm 6.04$ & $78.61 \pm 6.06$ & 2.030 & 0.155 \\
$\quad$ Range & $65.64-92.25$ & $69.06-90.96$ & $63.46-88.51$ & & \\
After anesthetic induction: & $66.69 \pm 5.20$ & $68.29 \pm 5.48$ & $65.21 \pm 6.04$ & 1.808 & 0.104 \\
$\quad$ Mean \pm SD & $54.56-74.09$ & $60.27-83.91$ & $54.71-72.46$ & & \\
$\quad$ Range & & & & & \\
Immediately after intubation: & $72.23 \pm 6.58$ & $83.73 \pm 6.42 \dagger$ & $85.76 \pm 7.51 \dagger$ & 8.308 & $<0.001^{* *}$ \\
$\quad$ Mean \pm SD & $58.94-84.37$ & $73.14-97.03$ & $64.1-101.94$ & & \\
$\quad$ Range & & & & & \\
At 1min.: & $73.63 \pm 7.40$ & $83.74 \pm 5.55 \dagger$ & $88.01 \pm 6.61 \dagger$ & 11.32 & $<0.001^{* *}$ \\
$\quad$ Mean \pm SD & $61.04-93.92$ & $70.31-94.81$ & $76.95-102.27$ & & \\
$\quad$ Range & & & & & \\
At 3min.: & $70.12 \pm 7.81$ & $75.25 \pm 6.60 \dagger$ & $75.24 \pm 5.83 \dagger$ & 4.729 & $0.012^{*}$ \\
$\quad$ Mean \pm SD & $58.2-86.14$ & $61.93-87.12$ & $62.06-86.6$ & & \\
$\quad$ Range & & & & & \\
At 5 min.: & $71.61 \pm 6.97$ & $75.16 \pm 4.97 \dagger$ & $76.31 \pm 6.53 \dagger$ & 3.887 & $0.025^{*}$ \\
$\quad$ Mean \pm SD & $58.9-83.22$ & $67.77-84.77$ & $63.23-87.71$ & & \\
$\quad$ Range & & & & & \\
\hline
\end{tabular}

This table shows statistically significant difference between groups according to mean arterial

blood pressure $(\mathrm{mmHg})$, immediately after intubation up to $5 \mathrm{~min}$.

Table (8): Comparison between groups according to heart rate (beat $/ \mathrm{min})$.

\begin{tabular}{|c|c|c|c|c|c|}
\hline Heart Rate (beat/min) & $\begin{array}{c}\text { Group A: } \\
\text { Magnesium sulphate } \\
(n=25)\end{array}$ & $\begin{array}{l}\text { Group B: } \\
\text { Ketamine } \\
(n=25)\end{array}$ & $\begin{array}{l}\text { Group C: } \\
\text { Rocuronium } \\
\quad(\mathrm{n}=25)\end{array}$ & ANOVA & $\begin{array}{c}p- \\
\text { value }\end{array}$ \\
\hline \multicolumn{6}{|l|}{ Baseline: } \\
\hline Mean \pm SD & $74.11 \pm 6.32$ & $76.72 \pm 7.90$ & $77.75 \pm 8.52$ & \multirow[t]{2}{*}{1.512} & \multirow[t]{2}{*}{0.227} \\
\hline Range & $60.47-84.9$ & $60.71-91.11$ & $62.46-101.14$ & & \\
\hline \multicolumn{6}{|c|}{ After anesthetic induction: } \\
\hline Mean \pm SD & $71.10 \pm 6.25$ & $72.58 \pm 5.08$ & $71.03 \pm 6.98$ & \multirow[t]{2}{*}{0.506} & \multirow[t]{2}{*}{0.605} \\
\hline Range & $61.91-84.98$ & $64.92-84.29$ & $55.23-79.98$ & & \\
\hline \multicolumn{6}{|c|}{ Immediately after intubation: } \\
\hline Mean \pm SD & $69.84 \pm 8.48$ & $70.38 \pm 8.16$ & $80.44 \pm 6.42 \dagger \dagger$ & \multirow[t]{2}{*}{14.858} & \multirow[t]{2}{*}{$<0.001 * *$} \\
\hline Range & $56.36-82.12$ & $53.91-87.95$ & $66.53-92.8$ & & \\
\hline \multicolumn{6}{|l|}{ At 1min.: } \\
\hline Mean \pm SD & $76.09 \pm 6.18$ & $76.38 \pm 7.62$ & $84.31 \pm 7.38 \dagger t$ & \multirow[t]{2}{*}{10.820} & \multirow[t]{2}{*}{$<0.001 * *$} \\
\hline Range & $64.56-94.65$ & $67.19-95.52$ & $65.79-98.12$ & & \\
\hline \multicolumn{6}{|l|}{ At $3 \min .:$} \\
\hline Mean \pm SD & $76.84 \pm 6.87$ & $77.48 \pm 5.72$ & $82.67 \pm 8.68 \dagger t$ & \multirow[t]{2}{*}{4.416} & \multirow[t]{2}{*}{$0.016^{*}$} \\
\hline Range & $62.31-92.35$ & $68.86-94.62$ & $62.34-94.68$ & & \\
\hline \multicolumn{6}{|l|}{ At 5min.: } \\
\hline Mean \pm SD & $78.00 \pm 5.72$ & $79.57 \pm 6.84$ & $83.62 \pm 5.83 \dagger t$ & \multirow[t]{2}{*}{2.251} & \multirow[t]{2}{*}{$0.013 *$} \\
\hline Range & $65.12-88.5$ & $64.73-92.55$ & $69.1-93.45$ & & \\
\hline
\end{tabular}

TOF ratio at time point of intubation $\%$

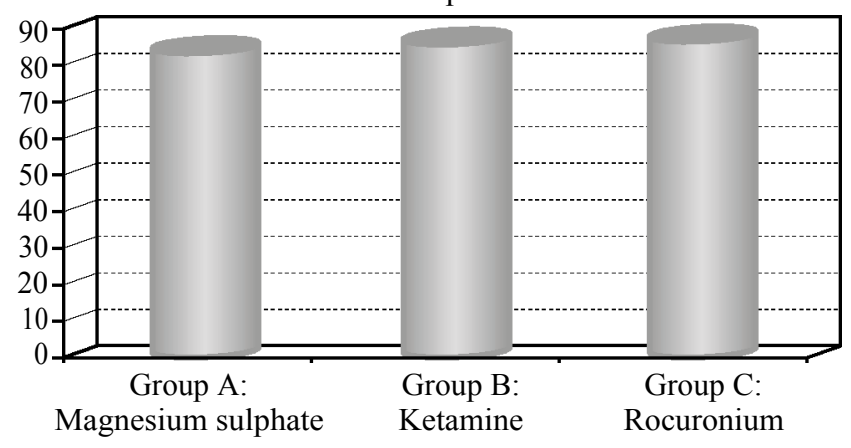

Fig. (8): Bar chart between groups according to TOF ratio at time point of intubation $\%$. 

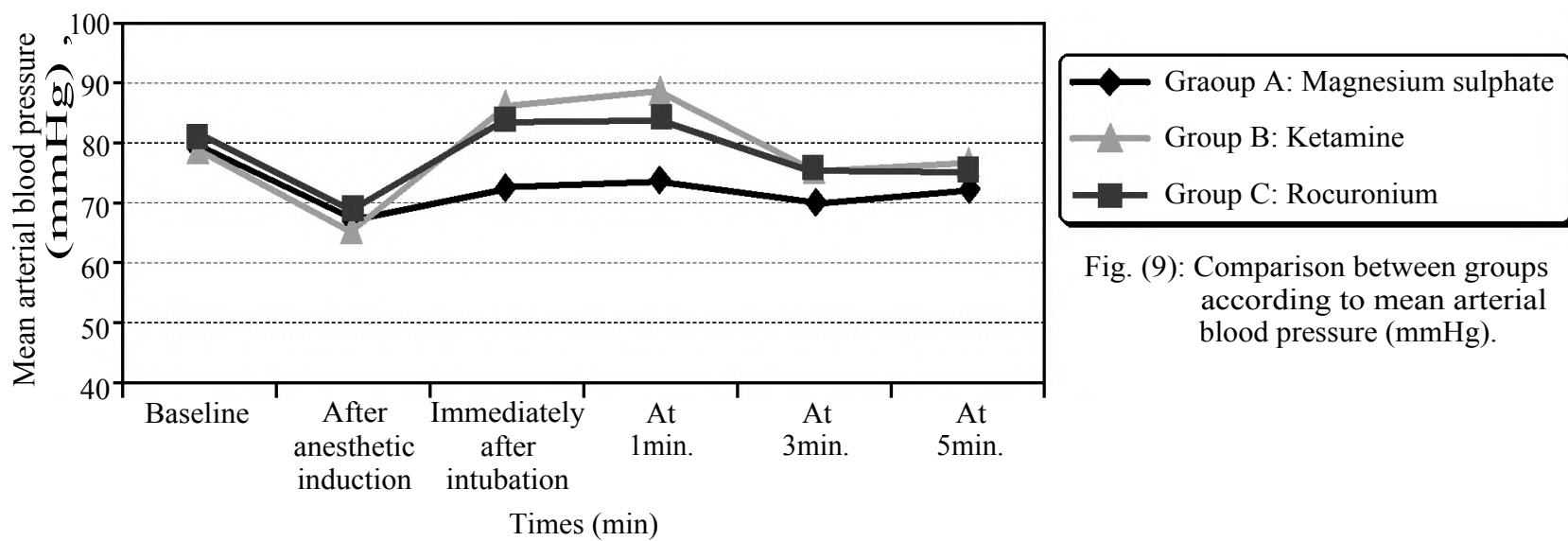

Fig. (9): Comparison between groups according to mean arterial blood pressure $(\mathrm{mmHg})$.

Table (9): Comparison between groups according to intubation condition.

\begin{tabular}{|c|c|c|c|c|c|}
\hline Intubating condition & $\begin{array}{c}\text { Group A: } \\
\text { Magnesium sulphate } \\
(\mathrm{n}=25)\end{array}$ & $\begin{array}{l}\text { Group B: } \\
\text { Ketamine } \\
(\mathrm{n}=25)\end{array}$ & $\begin{array}{l}\text { Group C: } \\
\text { Rocuronium } \\
(\mathrm{n}=25)\end{array}$ & $x^{2}$ & $\begin{array}{c}p- \\
\text { value }\end{array}$ \\
\hline $\begin{array}{l}\text { Laryngoscopy: } \\
\text { Jaw relaxation: } \\
\text { Relaxed }\end{array}$ & $25(100.0 \%)$ & $25(100.0 \%)$ & $25(100.0 \%)$ & 0.000 & 1.000 \\
\hline $\begin{array}{l}\text { Resistance to blade: } \\
\text { No resistance } \\
\text { Slight resistance }\end{array}$ & $\begin{array}{ll}25 & (100.0 \%) \\
0 & (0.0 \%)\end{array}$ & $\begin{array}{ll}25 & (100.0 \%) \\
0 & (0.0 \%)\end{array}$ & $\begin{array}{ll}0 & (0.0 \%) \\
25 & (100.0 \%)\end{array}$ & 150.000 & $<0.001 * *$ \\
\hline $\begin{array}{l}\text { Position of vocal cord: } \\
\text { Abducted } \\
\text { Intermediate }\end{array}$ & $\begin{array}{ll}25 & (100.0 \%) \\
0 & (0.0 \%)\end{array}$ & $\begin{array}{ll}0 & (0.0 \%) \\
25 & (100.0 \%)\end{array}$ & $\begin{array}{ll}0 & (0.0 \%) \\
25 & (100.0 \%)\end{array}$ & 75.000 & $<0.001 * *$ \\
\hline $\begin{array}{l}\text { Diaphragmatic movement or cough: } \\
\text { None }\end{array}$ & $25(100.0 \%)$ & $25(100.0 \%)$ & $25(100.0 \%)$ & 0.000 & 1.000 \\
\hline $\begin{array}{l}\text { Result: } \\
\quad \text { Excellent } \\
\text { Good }\end{array}$ & $\begin{array}{ll}25 & (100.0 \%) \\
0 & (0.0 \%)\end{array}$ & $\begin{array}{ll}0 & (0.0 \%) \\
25 & (100.0 \%)\end{array}$ & $\begin{array}{ll}0 & (0.0 \%) \\
25 & (100.0 \%)\end{array}$ & 75.000 & $<0.001^{* *}$ \\
\hline
\end{tabular}

Table (8) shows statistically significant difference between groups according to heart rate immediately after intubation up to $5 \mathrm{~min}$.
Table (9) shows statistically significant difference between groups according to resistance to blade, position of vocal cord and result.

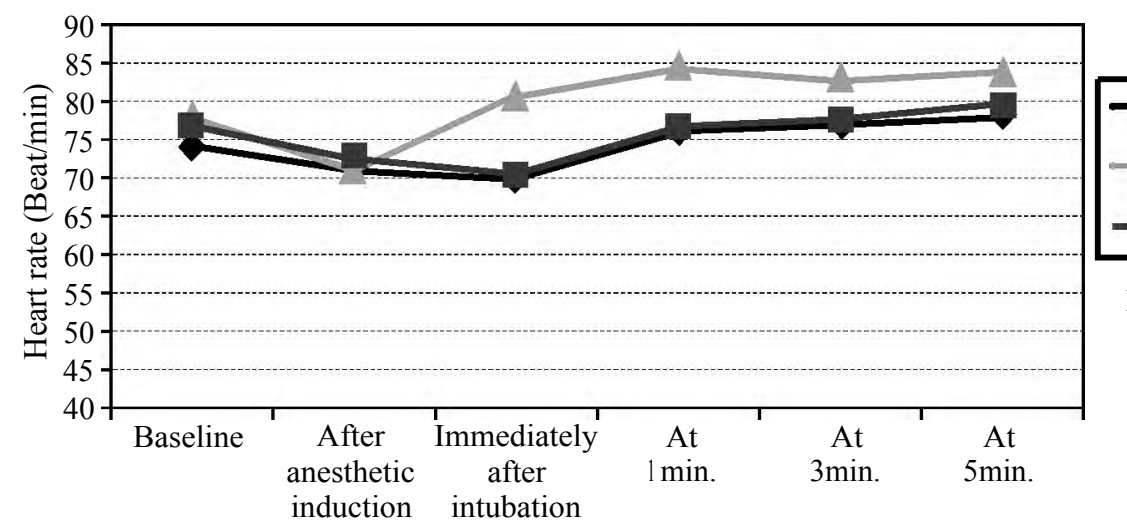

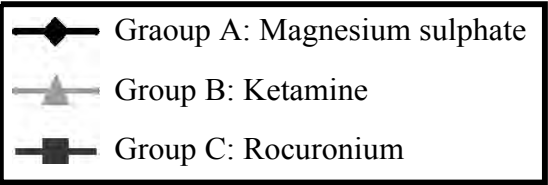

Fig. (10): Comparison between groups according to heart rate.

Times (min)

\section{Discussion}

The excellent intubating condition was more frequent in the magnesium group compared with the other groups (control, ketamine). Onset of neuromuscular block was shorter in the magnesium group than in the control and ketamine.
In the present study, magnesium sulphate pretreatment provided an excellent intubating condition for rapid sequence induction. Magnesium potentiates the effect of muscle relaxants mainly by decreasing presynaptic acetylcholine release [7]. The magnesium group had a $40 \%$ reduction in onset time compared with the controls, consistent with 
a previous study that showed a $36 \%$ reduction in rocuronium onset time by magnesium sulphate [4] Therefore, the rapid neuromuscular block may be the major contributing factor to the superior intubating condition in the magnesium group. Magnesium attenuates catecholamine release and elevation of blood pressure after intubation [8]. Although mean arterial pressure tended to be lower in the magnesium group, it was not statistically different among the groups in this study. This may be partially attributed to fentanil, which may have blunted cardiovascular changes in all groups [9]

Ketamine neither improved intubating condition nor accelerated rocuronium onset when compared with the control group, in contrast to a previous report [3]. It was postulated that increased cardiac output and blood pressure by ketamine would facilitate rocuronium delivery to the relevant neuromuscular cleft, leading to a faster neuromuscular block [3]. However, the blood pressure difference between ketamine and the other groups was smaller in the present study than in the previous report [3] presumably due to differences in induction agents and doses. The higher dose of propofol $(2 \mathrm{mg} / \mathrm{kg})$ used in the previous study may have induced more profound hypotension in the controls. In this regard, rocuronium onset may not be obviously shortened by ketamine in comparison with the control group if differences in cardiac output or blood pressure between the ketamine and control groups were attenuated by various factors such as the titrated dose of propofol.

Magnesium sulphate did not significantly prolong rocuronium duration compared with the control. This may be because the sample size was insufficient, or the magnesium sulphate dose $(50 \mathrm{mg} /$ $\mathrm{kg}$ ) may have been too small to increase rocuronium duration; another study with double the sample size and with a larger dose of magnesium sulphate $(60 \mathrm{mg} / \mathrm{kg})$ found that rocuronium duration was increased by magnesium sulphate [4]

In addition, the time lag between the magnesium sulphate and rocuronium injections in the present study may have attenuated the possible prolongation of rocuronium duration; magnesium delayed only the early recovery period but not the late phase in the previous study, which implies a diminishing effect of magnesium on rocuronium duration, as time goes on [4]. In addition, the 7-minute increase in duration by magnesium compared with the control (35 vs. 42 minutes) does not seem to have substantial clinical significance except for in very short procedures. Despite various methods of facilitating rapid-sequence intubation such as keta- mine pre-treatment clinicians can be confused about which method to choose without reference literature that demonstrates comparisons among the methods. Intubating condition based on magnesium pretreatment has not been compared with that based on ketamine pre-treatment.

Intubating condition can be influenced by numerous factors such as drugs, protocol, and clinician $[5,10]$. Therefore, direct objective comparisons should be made under standardized and equivalent settings. The protocol of this study followed the clinical research consensus [5] to produce reliable data. In this regard, this study may provide reference information when rapid-sequence intubation is needed. A potential limitation of this study is that conclusions about variables other than intubating condition cannot be drawn because the sample size was calculated only for the primary outcome. Further study is required to elucidate comparisons of such variables as onset and duration of rocuronium and hemodynamic variables among the groups. Another limitation is that the primary end point for the power calculation, namely, "excellent" intubating conditions is highly subjective. Nonetheless, it is a widely used intubation score [11].

In conclusion, magnesium sulphate pretreatment was most likely to provide excellent intubating condition for rapid-sequence intubation compared with the control, ketamine pre-treatment. However, magnesium sulphate administration is associated with a burning or heat sensation.

\section{References}

1- EL-ORBANY M. and CONNOLLY L.A.: Rapid sequence induction and intubation: Current controversy. Anesth. Analg., 110: 1318-25, 2010

2- NAGUIB M. and LIEN C.: Pharmacology of muscle relaxants and their antagonists. In: Miller RD, editor. Miller's Anesthesia. 7 th ed. Philadelphia: Churchill Livingstone Elsevier, p. 859-911, 2009.

3- TOPCUOGLU P.T., UZUN S., CANBAY O., PAMUK G. and OZGEN S.: Ketamine, but not priming, improves intubating conditions during a propofol rocuronium induction. Can. J. Anesth., 57: 113-9, 2010.

4- CZARNETZKI C., LYSAKOWSKI C., ELIA N. and TRAMER M.: Time course of rocuronium induced neuromuscular block after pre treatment with magnesium sulphate: A randomised study. Acta. Anaesthesiol. Scand, 54: 299-306, 2010

5- FUCHS BUDER T., CLAUDIUS C., SKOVGAARD L., ERIKSSON L., MIRAKHUR R. and VIBY MOGENSEN J.: Good clinical research practice in pharmacodynamics studies of neuromuscular blocking agents II: The Stockholm revision. Acta. Anaesthesiol. Scand., 51: 789-808, 2007. 
6- CORMACK R.S. and LEHANE J.: Difficult tracheal intubation in obstetrics. Anaesthesia, 39: 1105-11, 1984.

7- HERROEDER S., SCHONHERR M.E., De HERT S.G. and HOLLMANN M.W.: Magnesium-essentials for anesthesiologists. Anesthesiology, 114: 971-93, 2011.

8- JAMES M.F.M., BEER R.E. and ESSER J.D.: Intravenous magnesium sulphate inhibits catecholamine release associated with tracheal intubation. Anesth. Analg., 68: 7726, 1989.

9- GUIGNARD B., MENIGAUX C., DUPONT X., FLETCHER D. and CHAUVIN M.: The effect of remifen- tanil on the bispectral index change and hemodynamic responses after oro tracheal intubation. Anesth. Analg., 90: 161-7, 2000.

10- MAGORIAN T., FLANNERY K.B. and MILLER R.D.: Comparison of rocuronium, succinylcholine, and vecuronium for rapid-sequence induction of anesthesia in adult patients. Anesthesiology, 79: 913-8, 1993.

11- PERRY J.J., LEE J.S., SILLBERG V.A. and WELLS G.A.: Rocuronium versus succinylcholine for rapid sequence induction intubation. Cochrane Database Syst. Rev., 2: CD002788, 2008.

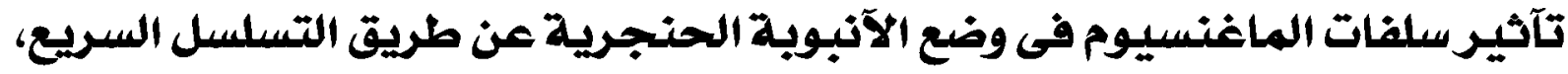

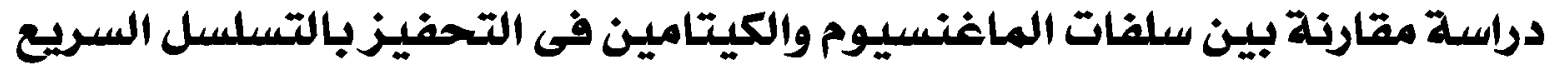

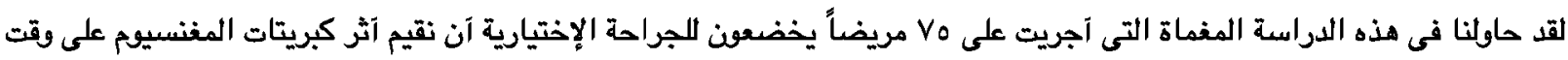 \\ البدء، ومدة ونسبة قطار الآربعة والعلامات العيوية وظروف التنبيب من بروميد الروكرونيوم. \\ ملخص النتائج التى توصلنا إليها هي حالة التنبيب الممتازة كانت آكثر تكراراً فى مجموعة المغنسيوم مقارنةً بالمجموعات الآخرى، وكان \\ إحصار الكتلة العصبية العضلية آقصر فى مجموعة المغنسيوم مما كان عليه فى مجموعات التحكم والكيتامين. \\ لم تعمل كبريتات المغنسيوم على إطالة مدة الروكورونيوم بشكل ملحوظ مقارنة مع التمكم. قد يكون هذا بسبب آن جرعة المغنسيوم

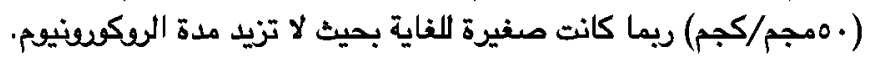

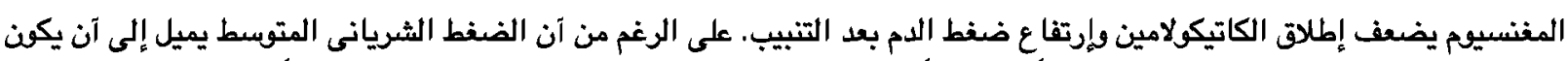

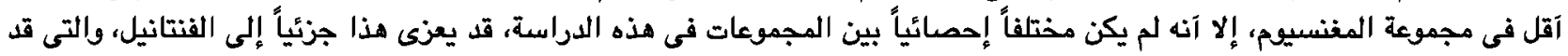 \\ تكون قد آضعفت تغيرات القلب والآوعية الدموية في جميع المجموعات.
}

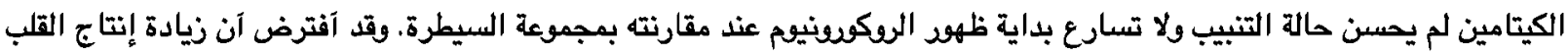

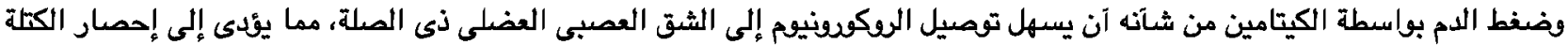
العصبية والعضلية بشكل آسرع. 